\title{
Developmental constraints in the Drosophila wing
}

\author{
DANIELA GUERRA, MARIA CRISTINA PEZZOLI, GIANFRANCO GIORGI, FLAVIO \\ GAROIA \& SANDRO CAVICCHI* \\ Dipartimento di Biologia evol. sper., Università di Bologna, via Selmi 3, 40126-Bologna, Italy
}

\begin{abstract}
Selection experiments for shortening the four longitudinal veins in a wild population of Drosophila melanogaster have been performed to evaluate how a local change is integrated in the wing development. Our results show that, though many units of selection seem to exist within a given organ, these are strongly constrained within the developmental programme, in such a way that only some predictable forms are expected. The results are discussed in terms of the 'Entelechia' model proposed by Garcia-Bellido in which the intercalarity of positional values promoted by 'martial' genes in a given organ is the driving force for controlled cell proliferation.
\end{abstract}

Keywords: artificial selection, developmental constraints, Drosophila melanogaster.

\section{Introduction}

The process building up the size and shape of body parts in higher organisms involves spatially and temporally co-ordinated cell proliferation. The evolution of the growth pattern appears to be greatly constrained; this could depend either on the homeostatic organization of the genetic system (Lerner, 1954; Carson, 1975) or upon the inflexibility of the developmental system.

Our studies on this topic have shown that the developmental mechanism underlying the wing development of Drosophila melanogaster can be modulated. We have found that the Anterior and Posterior wing compartments, which are independent subunits of development (Garcia-Bellido et al., 1973), also behave as units of selection. They contribute differentially to wing size of populations evolved at different temperatures and their variation has an adaptive significance (Cavicchi et al., 1978, 1985, 1991). In spite of that, only minor allometric variation can be found after natural selection in the laboratory (Cavicchi et al., 1985, 1991) or in the wild (Weber, 1990). The most straightforward explanation is that wing morphology is the result of continuous natural selection around a local optimum. This seems to be confirmed by the fact that artificial

\footnotetext{
${ }^{*}$ Correspondence. E-mail: cavicchi_s@ biblio.cib.unibo.it
}

selection on wing allometry induces a very rapid response (Weber, 1990), suggesting that much additive genetic variance exists for individual dimensions within the wing. On these bases, the developmental pattern can be changed in any direction by selection.

Further selection experiments have shown that very small regions can respond to selection almost independently (Weber, 1992), suggesting that the control of wing pattern formation must involve many genes and that many units of selection, other than major wing compartments, can exist. Recent experiments in developmental genetics seem to support this idea. The growing wing imaginal disc appears as a mosaic of cell proliferation centres (GonzalesGaitan et al., 1994) located within the major wing compartments $\quad$ (Anterior $=\mathrm{A}, \quad$ Posterior $=\mathrm{P}$, Dorsal $=\mathrm{D}$ and Ventral $=\mathrm{V}$ ) which are restricted to the areas giving rise to the intervein regions of the adult wing. The presumptive vein regions represent distinct boundaries which delimit the proliferation centres to the intervein regions (Garcia-Bellido \& de Celis, 1992; Garcia-Bellido et al., 1994; GonzalesGaitan et al., 1994).

A generative model that has been proposed (Garcia-Bellido \& de Celis, 1992; Garcia-Bellido et al., 1994; Gonzales-Gaitan et al., 1994) suggests that the control of cell proliferation is the result of cell interactions. Neighbouring cells exchange quantitative signals communicating positional values that 
reflect the amount of gene activity of so-called 'martial' genes. Heterogeneities in the distribution of cells with higher martial function, e.g. compartment boundaries or veins, would be communicated to other cells (via ligands/activated receptors) and thus elicit their mitotic division when their own martial values are lower than those of the signalling cell. Cell proliferation will cease and the 'Entelechia condition' be reached when the differences in values between neighbouring cells are indistinguishable in cell-cell signalling. On these bases, intervein regions can be considered as further subunits of development. Their interaction during wing morphogenesis could explain the high developmental homeostasis always found for wing size and shape.

The size of a very small morphological domain can be altered by selection with a small correlated response of the surrounding regions (Weber, 1992), indicating that there are no apparent limits to morphological evolution. In the present work, by selecting for small size of the four longitudinal veins of the Drosophila wing, we evaluate how a local change is integrated in development, providing insight into the way in which the pattern can evolve.

\section{Materials and methods}

The selection experiment was performed on a wildtype strain captured in central Italy (Rieti). Flies were maintained for about one year at a constant temperature of $25^{\circ} \mathrm{C}$ on standard medium as a mass population. Two replicates (R1 and R2) of 100 pairs were sampled from the mass population and each pair was allowed to lay eggs for three days, changing the vials each day to avoid crowding. At eclosion, more than 500 virgin females and males were collected, mixed and then distributed into five groups for each replicate. Each group represents the base line of each selection and its control. After measurements under a binocular microscope, at a total magnification of $24.8 \times$ with a graduated scale of $1 \mathrm{~mm}$ subdivided into 100 parts placed in an ocular, the 10 pairs showing the smallest vein size (L2, L3, L4 or L5) were chosen as parents for the following generation. We selected only in the minus direction as, from the literature (Falconer, 1981 for a review), selection for wing size shows an asymmetric response that is greater in the minus direction. Our selection continued following the same procedure (selection intensity $=0.1$ ) for 10 generations in replicate 1 and 11 in replicate 2 . Then we released all the lines by mass rearing in larger bottles for a further 10 generations. The two replicates, with their unselected controls, were main-

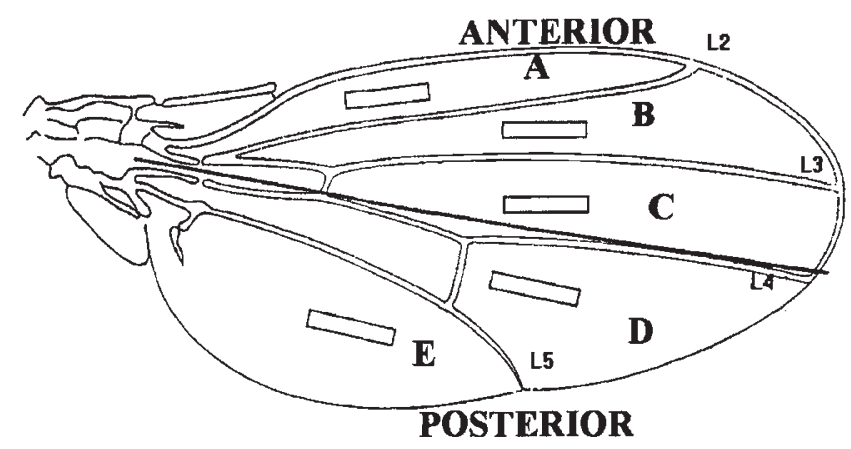

Fig. 1 Schematic representation of the adult wing of Drosophila melanogaster. L2-L5 longitudinal veins; A-E intervein regions; rectangles mark the surfaces in which cells were counted.

tained in two different thermostatic cells but, owing to a sudden increase in temperature during the release, one of these was lost.

To estimate the correlated response of unselected vein lengths and intervein regions, the right wings of 50 females for each line of only one replicate were pulled out and mounted on slides at the 10th generation of selection as well as at the 10th released generation.

The areas of different intervein regions (Fig. 1) were measured by computer on the basis of the number of pixels included in a given intervein region. For this purpose the MTV3 program provided by Data Crunch (S. Clemente, CA) was used. The boundary between the Anterior and Posterior compartments lies just anteriorly to the $\mathrm{L} A$ vein. We approximated the compartment areas, taking the sum of $A, B$ and $C$ or of the $D$ and $E$ regions as representative of the Anterior or Posterior compartments, respectively (Cavicchi et al., 1985, 1991).

Cell size and number were also estimated in the dorsal blade of different regions of the selected lines and of the unselected control. The hairs included in a surface of $0.009 \mathrm{~mm}^{2}$ were counted (Fig. 1). Cell area was obtained by dividing the surface by the number of hairs. Cell number was obtained by dividing the surface of the intervein region by cell area. As cell density changes in different regions, we took care to place the reticule always in the same position, taking crossveins as reference points as far as possible from the veins.

\section{Results}

Figure 2 gives the standardized response of the four selected lines for the two replicates. The selection for decreasing lengths is more efficient on L3 and 
L4 than on L2 and L5 veins and this is reflected in higher $h^{2}$ estimates (Table 1).

Table 2 gives Dunnett's $t$-values for the differences from the unselected control of wing vein lengths and areas of different regions at the 10th generation of selection. Each line shows different responses: in general, with the sole exception of the L4 selection, the selected trait shows the greater response. However, the correlation between traits did not change during selection and remained high and constant $(r>0.6)$ in all lines. Decreases in the surface areas of the intervein regions were evident for all selected lines with the sole exception of the D region in the $\mathbf{L} 5$ selection.

The relative contribution of each region is given (intervein area/total area) as Dunnett's $t$-values for the differences from the unselected line in Table 3. Positive values indicate a larger contribution of a given region to decreasing wing size, and vice versa for the negative ones. Different regions behaved as different units of response to selection, but strongly interacting. Their contribution changed according to the selected line, but, in general, it was positive for the regions located posteriorly and negative for those located anteriorly to the selected vein. The largest contribution was provided.by the $\mathrm{E}$ region in all lines and by the $\mathrm{C}$ region in $\mathrm{L} 2$ and $\mathrm{L} 3$ selections. The only exception was the A region in the L5 selection. Interestingly, the magnitude of the correlated response of the $\mathrm{E}$ region increased when the distance from the selected vein decreased.

As a consequence, the surface areas of the two major compartments, Anterior and Posterior, appeared to contribute in a similar fashion when selection was performed on veins belonging to the Anterior compartment (L2 or L3), but the Posterior
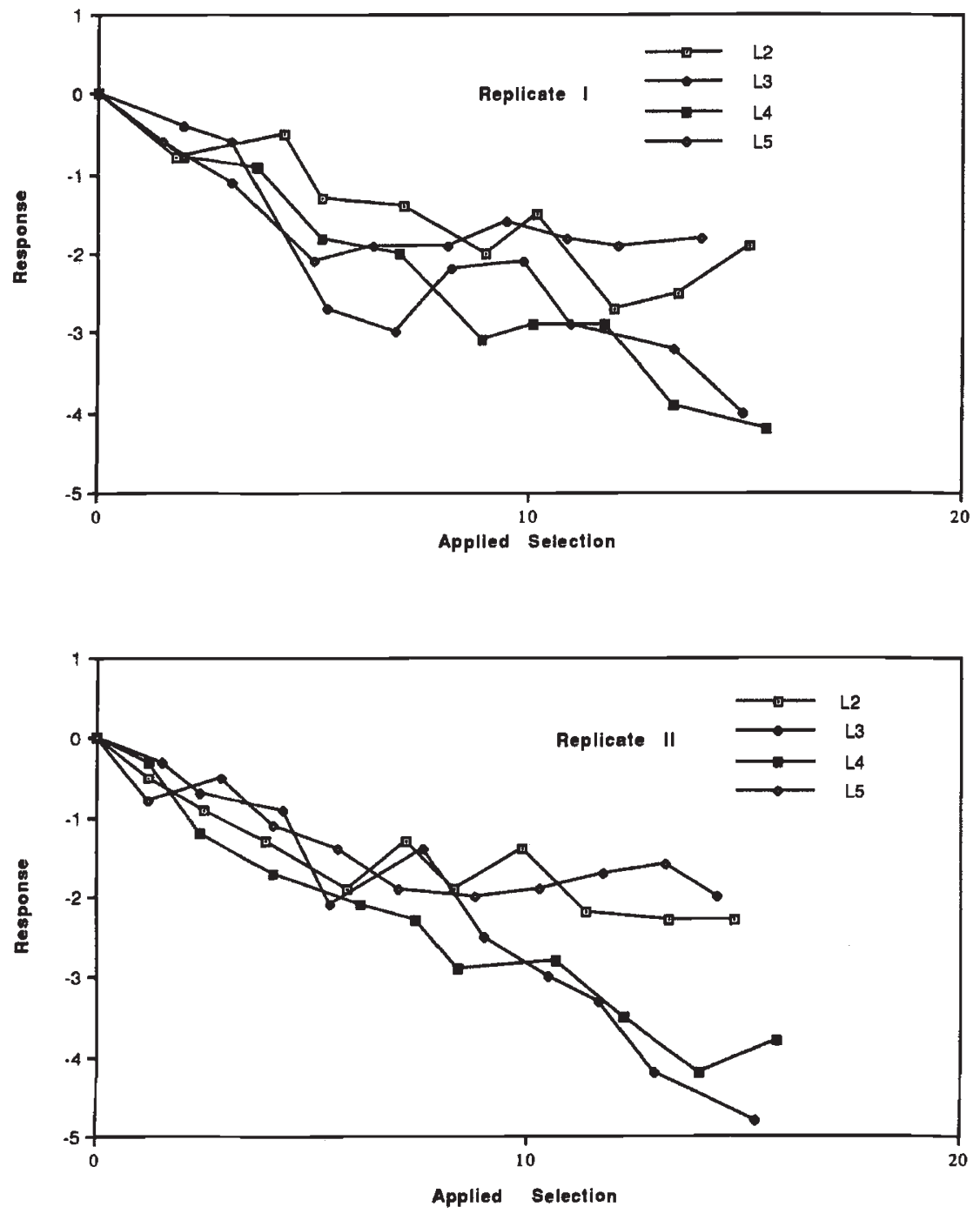

Fig. 2 Plot of standardized response on standardized applied selection for short wing veins in the two replicates of Drosophila melanogaster. 
Table $1 h^{2}$ values estimated on the basis of the standardized response to the standardized applied selection in the four selected lines of Drosophila melanogaster

\begin{tabular}{lcccc}
\hline & \multicolumn{4}{c}{ Selected lines } \\
\cline { 2 - 5 } Replicate & L2 & L3 & L4 & L5 \\
\hline R1 & 0.153 & 0.225 & 0.269 & 0.110 \\
& $(0.029)$ & $(0.042)$ & $(0.021)$ & $(0.034)$ \\
R2 & 0.148 & 0.271 & 0.255 & 0.123 \\
& $(0.023)$ & $(0.027)$ & $(0.015)$ & $(0.029)$ \\
\hline
\end{tabular}

Standard errors in parentheses.

compartment contributed more when the selection was performed on the L4 and L5 veins (Anterior/ total area).

Differences from the control in cell area and number are given for each region in Table 4. The overall count gives $\approx 10500$ cells in the dorsal wing blade of the control. Different intervein regions seem to possess different mechanisms for responding to artificial selection. The $\mathrm{A}$ and more so the $\mathrm{C}$ and $D$ regions reduced the surface on the basis of cell area, whereas the $B$ and $E$ regions reduced it according to cell number, irrespective of the selected

Table 2 Two-sided comparisons (Dunnett's $t$ ) between the unselected control Drosophila melanogaster and the four selected lines for wing vein length and areas of intervein regions, Anterior and Posterior compartments and total wing (only Replicate 1)

\begin{tabular}{lrrrc}
\hline & \multicolumn{4}{c}{ Selected lines } \\
\cline { 2 - 5 } $\begin{array}{l}\text { Veins and } \\
\text { wing regions }\end{array}$ & \multicolumn{1}{c}{ L2 } & L3 & L4 & L5 \\
\hline L2 & 11.61 & 16.04 & 11.56 & 5.93 \\
L3 & 9.54 & 17.86 & 14.04 & 5.24 \\
L4 & 9.32 & 16.56 & 12.18 & 4.82 \\
L5 & 10.11 & 16.46 & 11.96 & 7.91 \\
A & 4.43 & 14.79 & 5.31 & 4.11 \\
B & 6.92 & 14.88 & 4.27 & 3.21 \\
C & 8.54 & 17.39 & 7.39 & 2.71 \\
Anterior & 7.34 & 16.71 & 6.30 & 3.52 \\
D & 6.21 & 15.46 & 5.90 & 1.82 NS \\
E & 7.72 & 15.22 & 8.48 & 6.21 \\
Posterior & 7.54 & 15.65 & 7.70 & 4.41 \\
Total wing & 7.65 & 16.54 & 7.29 & 4.08 \\
\hline
\end{tabular}

Comparisons are based on 50 wings per line. When not specified, all values are highly significant $(P<0.01)$. NS not significant.
Table 3 Two-sided comparisons (Dunnett's $t$ ) between the unselected control Drosophila melanogaster and the four selected lines for the ratios of the intervein regions and Anterior and Posterior compartments to total wing area (only Replicate 1)

\begin{tabular}{lcccc}
\hline \multirow{2}{*}{$\begin{array}{l}\text { Ratios to } \\
\text { total wing area }\end{array}$} & L2 & L3 & L4 & L5 \\
\cline { 2 - 5 } & & & & \\
\hline A & $-3.31^{* *}$ & -0.27 & -0.12 & 2.11 \\
B & -0.66 & $-4.20^{* *}$ & $-3.72^{* *}$ & -2.12 \\
C & 2.16 & $3.33^{* *}$ & -0.21 & $-4.11^{* *}$ \\
Anterior & -1.04 & -0.71 & $-2.83^{*}$ & $-2.59^{*}$ \\
D & -0.87 & -1.87 & -0.63 & $-5.01^{* *}$ \\
E & 2.01 & $2.48^{*}$ & $3.48^{* *}$ & $7.21^{* *}$ \\
Posterior & 1.04 & 0.71 & $2.83^{*}$ & $2.59^{*}$ \\
\hline
\end{tabular}

Comparisons are based on 50 wings per line.

${ }^{*} P<0.05,{ }^{*} P<0.01$.

trait. The prevailing overall effect (total wing) was a decreasing cell number in all selections with a joint effect of cell area in the Anterior compartment and in the Posterior one of the L3 selection. Interestingly, in almost all the regions of the L4 and L5 selections when cell area (or number) decreased, cell number (or area) increased.

A high positive correlation was present between the surface area of each region and cell number $(r>0.7)$ and a negative correlation between cell size and number $(r>-0.6)$, but no correlation was observed between intervein surface and cell size in all selected lines and in the control, supporting the above behaviour at the population level.

Two of the released lines showed different behaviour from the other two irrespective of the magnitude of the realized responses to selection (Table 5). The L2 and L3 lines still maintained significant differences from the control both for vein lengths and intervein surfaces, whereas, on average, the remaining two lines (L4 and L5) reached size values similar to the control. In spite of this, the relative contributions of different intervein regions to total wing area were almost the same for all lines (Table 6). With the exception of only one significant effect (region $B$ in the L3 release), all the regions of the Anterior compartment (A, B and C) showed positive values, whereas those of the Posterior compartment ( $\mathrm{D}$ and $\mathrm{E}$ ) were negative. This is obviously reflected in positive values for the Anterior compartment. The general picture was that of released lines with different wing sizes but very 
similar wing shapes, in which the contribution of the Anterior compartment to the total wing area prevailed.

\section{Discussion}

In the present work we show that, although many units of selection seem to exist within a given organ, these are strongly constrained within the developmental programme, in such a way that only some predictable forms are expected.

When selection is performed on the longest veins (L3 and L4) the greatest response can be achieved. But if the quantity of response is similar in two-bytwo comparisons (L3, L4 and L2, L5 selections), the quality, i.e. the correlated response of different veins and regions, is not the same. This means that wing shape, and therefore the way in which wing blade grows and develops, is differently affected by the positional information associated with the selected trait.

Clonal analyses have shown that the wing blade is a mosaic of regions whose cells show independent proliferation control restricted by the presumptive vein regions. Thus, the presumptive veins are local symmetry axes (restriction borders) of cell proliferation within the major compartments (Garcia-Bellido \& de Celis, 1992; Garcia-Bellido et al., 1994; Gonzales-Gaitan et al., 1994).

The wing blade appears to be not only a spatial but also a temporal mosaic of cell proliferation centres (Gonzales-Gaitan et al., 1994; Milan et al., 1996). The regions located in the Anterior and Dorsal compartments appear to initiate cell proliferation before those located in the Posterior and Ventral ones. A consequence of this could be that the 'power' of martial values associated to veins is temporally scaled along the same direction.

Artificial selection decreasing the length of a given vein would decrease its martial value and induce an accommodation of the surrounding territories. The accommodation would follow both anterior-posterior and proximal-distal directions if the time at which cell proliferation takes place in the surrounding regions is the same or delayed. But the accommodation effect could also be lower if proliferation takes place earlier. The power of referring borders other than veins, represented by A-P

Table 4 Differences between the unselected control Drosophila melanogaster and the four selected lines in cell area $(a)$ and cell number $(n)$ of intervein regions, Anterior and Posterior compartments and the total wing (only Replicate 1)

\begin{tabular}{lccccc}
\hline & & \multicolumn{4}{c}{ Selected lines } \\
\cline { 2 - 6 } Wing & & $\mathrm{L} 2$ & $\mathrm{~L} 3$ & $\mathrm{~L} 4$ & $\mathrm{~L} 5$ \\
\hline $\mathrm{A}$ & & & & & \\
& & 0.0001 & $0.0040^{* *}$ & $0.0005^{*}$ & $0.0013^{* *}$ \\
$\mathrm{~B}$ & $n$ & 11 & 6 & $65^{* *}$ & 14 \\
& $a$ & $0.0005^{* *}$ & 0.0001 & -0.0002 & -0.0001 \\
$\mathrm{C}$ & $n$ & 31 & $344^{* *}$ & $148^{* *}$ & $136^{* *}$ \\
& $a$ & $0.0008^{* *}$ & $0.0028^{* *}$ & $0.0013^{* *}$ & $0.0005^{*}$ \\
Anterior & $n$ & 32 & $105^{*}$ & $-63^{*}$ & $80^{*}$ \\
& $a$ & $0.0005^{*}$ & $0.0023^{* *}$ & $0.0005^{*}$ & $0.0005^{*}$ \\
D & $n$ & 74 & $455^{* *}$ & $150^{*}$ & $229^{*}$ \\
& $a$ & $0.0004^{*}$ & $0.0016^{* *}$ & $0.0009^{* *}$ & $0.0010^{* *}$ \\
E & $n$ & 26 & $282^{* *}$ & 4 & -61 \\
& $a$ & 0.0000 & $0.0013^{* *}$ & -0.0006 & $-0.0011^{* *}$ \\
Posterior & $n$ & $158^{*}$ & $368^{* *}$ & $272^{* *}$ & $449^{* *}$ \\
& $a$ & 0.0002 & $0.0022^{* *}$ & 0.0002 & 0.0000 \\
Total wing & $a$ & 0.0003 & $0.0023^{* *}$ & 0.0003 & 0.0003 \\
& $n$ & $249^{*}$ & $1106^{* *}$ & $428^{* *}$ & $617^{* *}$ \\
\hline
\end{tabular}

Comparisons are based on 10 wings per line (Dunnett's $t$ test).

${ }^{*} P<0.05,{ }^{* *} P<0.01$. 
Table 5 Two-sided comparisons (Dunnett's $t$ ) between the unselected control Drosophila melanogaster and the four released lines for wing vein length and areas of intervein regions, Anterior and Posterior compartments and the total wing (only Replicate 1)

\begin{tabular}{|c|c|c|c|c|}
\hline \multirow{2}{*}{$\begin{array}{l}\text { Veins and } \\
\text { wing regions }\end{array}$} & \multicolumn{4}{|c|}{ Released lines } \\
\hline & $\mathrm{L} 2$ & L3 & L4 & L5 \\
\hline L2 & $4.72^{* *}$ & $8.01^{* *}$ & $3.63^{* *}$ & 0.74 \\
\hline L3 & $5.75^{* *}$ & $10.06^{* *}$ & $5.11^{* *}$ & 0.90 \\
\hline L4 & $4.84^{* *}$ & $8.00^{* *}$ & 2.46 & -0.98 \\
\hline L5 & $2.91^{* *}$ & $6.53^{* *}$ & 0.13 & -1.01 \\
\hline A & $4.08^{* *}$ & $11.25^{* *}$ & 1.16 & 2.49 \\
\hline B & $4.21^{* *}$ & $8.08^{* *}$ & 0.41 & 0.53 \\
\hline $\mathrm{C}$ & $4.54^{* *}$ & $9.27^{* *}$ & 0.12 & 0.26 \\
\hline Anterior & $4.49^{* *}$ & $9.77^{* *}$ & 0.53 & 0.97 \\
\hline $\mathrm{D}$ & $2.64^{*}$ & $6.46^{* *}$ & -0.81 & -1.68 \\
\hline $\mathrm{E}$ & 2.36 & $7.25^{* *}$ & -1.60 & -0.63 \\
\hline Posterior & $2.53^{*}$ & $6.98^{* *}$ & -1.25 & -1.15 \\
\hline Total wing & $3.52^{* *}$ & $8.34^{* *}$ & -0.42 & -0.16 \\
\hline
\end{tabular}

Comparisons are based on 50 wings per line.

${ }^{*} P<0.05,{ }^{* *} P<0.01$.

and $\mathrm{D}-\mathrm{V}$ boundaries, would also be relevant in this context.

In our experiment, the correlated response of various intervein regions, when the size of a given vein is reduced, seems to follow an A-P gradient with some partial regional discontinuity. In general, we found that the correlated response to any selected trait is larger for the regions located pos-

Table 6 Two-sided comparisons (Dunnett's $t$ ) between the unselected control Drosophila melanogaster and the four released lines for the ratios of the intervein regions and Anterior and Posterior compartments to total wing area (only Replicate 1)

\begin{tabular}{lcccc}
\hline \multirow{2}{*}{$\begin{array}{l}\text { Ratios to } \\
\text { total wing area }\end{array}$} & \multicolumn{4}{c}{ Leleased lines } \\
\cline { 2 - 5 } & L2 & L3 & L4 & L5 \\
\hline A & 2.22 & $5.43^{* *}$ & $3.81^{* *}$ & $6.15^{* *}$ \\
B & 1.48 & $-3.14^{* *}$ & 1.75 & 1.67 \\
C & $4.58^{* *}$ & $3.29^{* *}$ & $2.23^{*}$ & 1.69 \\
Anterior & $5.39^{* *}$ & $2.79^{*}$ & $5.14^{* *}$ & $5.81^{* *}$ \\
D & $-3.83^{* *}$ & $-7.62^{* *}$ & -1.75 & $-6.85^{* *}$ \\
E & $-3.58^{* *}$ & 1.75 & $-4.36^{* *}$ & -1.81 \\
Posterior & $-5.39^{* *}$ & $-2.79^{* *}$ & $-5.14^{* *}$ & $-5.81^{* *}$ \\
\hline
\end{tabular}

Comparisons are based on 50 wings per line.

${ }^{*} P<0.05,{ }^{*} P<0.01$. teriorly than for those located anteriorly. This is particularly evident when whole major compartments ( $\mathrm{A}$ and $\mathrm{P}$ ) are considered. In this case, both contribute similarly to the size of the wing blade when selection is performed on veins belonging to the A compartment, but the $\mathrm{P}$ one contributes less to total area (relative higher response) when the selection is performed on veins lying posteriorly.

The differential response of the two compartments seems to be a peculiarity of the developmental system. It is evident in mosaic analysis when clones homozygous for genes affecting cell proliferation ( $v n$ and/or $v e$ ) are induced in different regions (Garcia-Bellido et al., 1994), or in artificial (L4 vein, Cavicchi et al., 1981) or natural (Cavicchi et al., $1985,1991)$ selection experiments in which the $\mathrm{P}$ compartment appears to be the one with the largest genetic flexibility.

Within a compartment, the most posterior regions ( $\mathrm{C}$ in the Anterior and $\mathrm{E}$ in the Posterior compartment) were those showing the largest accommodation properties that decreased with the distance from the selected trait (Table 3). All these findings support the hypothesis that martial values are temporally scaled along the anterior-posterior direction and that some discontinuity at the A-P border exists. Interestingly, though the 'power' of the positional values associated to veins seemed to be changed by selection, the correlation between intervein surfaces remained high and unaffected in all selected lines $(r>0.7)$.

There seems to be a poor relationship between the magnitude of the response to artificial selection and the general outcome in terms of wing shape variation. The power of the change, which is also indicated when natural selection built up a new developmental equilibrium (released lines), seems to depend on the positional properties of the selected traits. Irrespective of the magnitude of the response, the size effects induced by selection on the anterior veins (L2 and L3) are retained after 10 release generations. Interestingly, all the released lines showed almost the same wing shape and the effect of the previous selections was only detectable on the A compartment. We have only one replicate of the released selection, but the probability that four independent lines reach the same shape equilibrium by chance seems very low. On these bases and also considering the different buffering properties of the two compartments, we argue that the genes responsible for size variation of the $\mathrm{P}$ compartment are hypostatic to those controlling the A one, so that the genetic change (direct or correlated), induced by previous artificial selection, is only maintained by 
the A compartment, which possesses the driving properties.

Selection for decreasing wing size induces, on the average, a reduction in cell number. This has already been well documented in previous experiments (Robertson, 1959b; Cavicchi, unpublished) and is also expected on the basis of the high correlation existing between wing (region) size and cell number that is always found in artificial and natural populations (Robertson, 1959a,b; Cavicchi et al., 1985 and in the present work). Again, different intervein regions appear to be units of size control with specific accommodation. There are regions showing a direct (or correlated) response to selection on the basis of cell area (A, C and D), and others on the basis of cell number ( $B$ and $E$ ). When selection is performed on veins belonging to the $\mathrm{P}$ compartment, in many regions, when cell area (number) decreases cell number (area) increases, providing further evidence of the buffering properties of the Posterior compartment.

The area of each region is, within a line, never correlated with cell area, although a high negative correlation between cell size and number is always present. We can consider this behaviour as an internal mechanism for body size stability probably related to the cell cycle: the faster cells grow, the smaller they would be. But this mechanism is also present at the population level: if one alters cell size by changing the rearing temperature (Cavicchi et al., 1985) or by selection (Robertson, 1959a and in the present work), cell number adjusts as if to compensate, at least partially, for wing size variation. In contrast, when selection involves cell number directly (C. Pezzoli, unpublished) or indirectly (Robertson, 1959b and present results), cell size changes to compensate cell number variation. The integration of these two variables at the phenotypic level is difficult to understand and could be explained, generically, as a homeostatic mechanism of the developmental system. Each of these is a selectable trait with a certain autonomy, and both are differently responsible for wing size variation as a consequence of either artificial or natural selection.

Our results show that the two cell variables, size and number, have different impacts on the areas of different intervein regions. The 'Entelechia model' suggests that the areas of intervein regions are determined by cell proliferation as a result of the intercalarity of martial values. This is only in part considered in our experiment where the changing of martial values was obtained by selection for wing size and not by precise changes of the growing properties of cells. In general, when the whole size is considered, our results are in good agreement with the model.

Our findings confirm that a given population possesses sufficient genetic resources to change the basic size and shape in many directions. Anyway, the basic developmental mechanisms underlying the morphogenetic process seem to be able to constrain the outcome of selection into some predictable form. The evolutionary consequence is that, unless mutation of major genes responsible for the basic architecture occurs, very few forms are expected within a species.

\section{Acknowledgements}

The authors are grateful to A. Garcia-Bellido and to all the people of his laboratory for valuable discussions and suggestions. The work was funded by MURST.

\section{References}

CARSON, H. L. 1975. The genetics of speciation at the diploid level. Am. Nat., 109, 83-92.

CAVICCHl, S., GIORGl, G. AND MOCHl, M. 1978. Investigations on early divergence between populations of Drosophila melanogaster kept at different temperatures. Genetica, 48, 81-87.

CAVICCHI, S., PEZZOLI, C. AND GIORGI, G. 1981. Correlation between characters as related to developmental pattern in Drosophila. Genetica, 56, 189-195.

CAVICCHI, S., GUERRA, D., GIORGI, G. AND PEZZoLI, C. 1985. Temperature-related divergence in experimental populations of Drosophila melanogaster. I. Genetic and developmental basis of wing size and shape variation. Genetics, 109, 665-689.

CAVICCHI, S., GIORGl, G., NATALI, V. AND GuerRA, D. 1991. Temperature-related divergence in experimental populations of Drosophila melanogaster. III. Fourier and centroid analysis of wing shape and relationship between shape variation and fitness. J. Evol. Biol., 4, $141-159$.

GARCla-BEllido, A. AND DE CELis, J. F. 1992. Developmental genetics of the venation pattern of Drosophila. Ann. Rev. Genet., 26, 277-304.

gARCla-bellido, A., Ripoll, P. AND morata, G. 1973. Developmental compartmentalisation of the wing disc of Drosophila. Nature New Biol., 245, 251-253.

GARCIA-BEllido, A., CORTES, F. AND Milan, M. 1994. Cell interactions in the control of size in Drosophila wings. Proc. Natl. Acad. Sci. U.S.A., 91, 10222-10226.

gonZales-gaitan, M. A., CAPDEVILA, M. P. AND GarciaBELlido, A. 1994. Cell proliferation patterns in the wing imaginal disc of Drosophila. Mech. Dev., 40, 183-200.

LeRner, I. M. 1954. Genetic Homeostasis. Dover Publications, New York.

(C) The Genetical Society of Great Britain, Heredity, 79, 564-571. 
MILAN, M., CAMPUZANO, s. AND GARCIA-BEllido, A. 1996. Cell cycling and patterned cell proliferation in the wing primordium of Drosophila. Proc. Natl. Acad. Sci. U.S.A., 93, 640-645.

ROBERTSON, F. W. 1959a. Studies in quantitative inheritance. XII. Cell size and number in relation to genetic and environmental variation of body size in Drosophila. Genetics, 44, 869-896.
ROBERTSON, F. W. 1959b. Studies in quantitative inheritance. XIII. Interrelation between genetic behavior and development in the cellular constitution of the Drosophila wing. Genetics, 44, 1113-1130.

WEBER, K. E. 1990. Selection on wing allometry in Drosophila melanogaster. Genetics, 126, 975-989.

WEBER, K. E. 1992. How small are the smallest selectable domains of form? Genetics, 130, 345-353. 